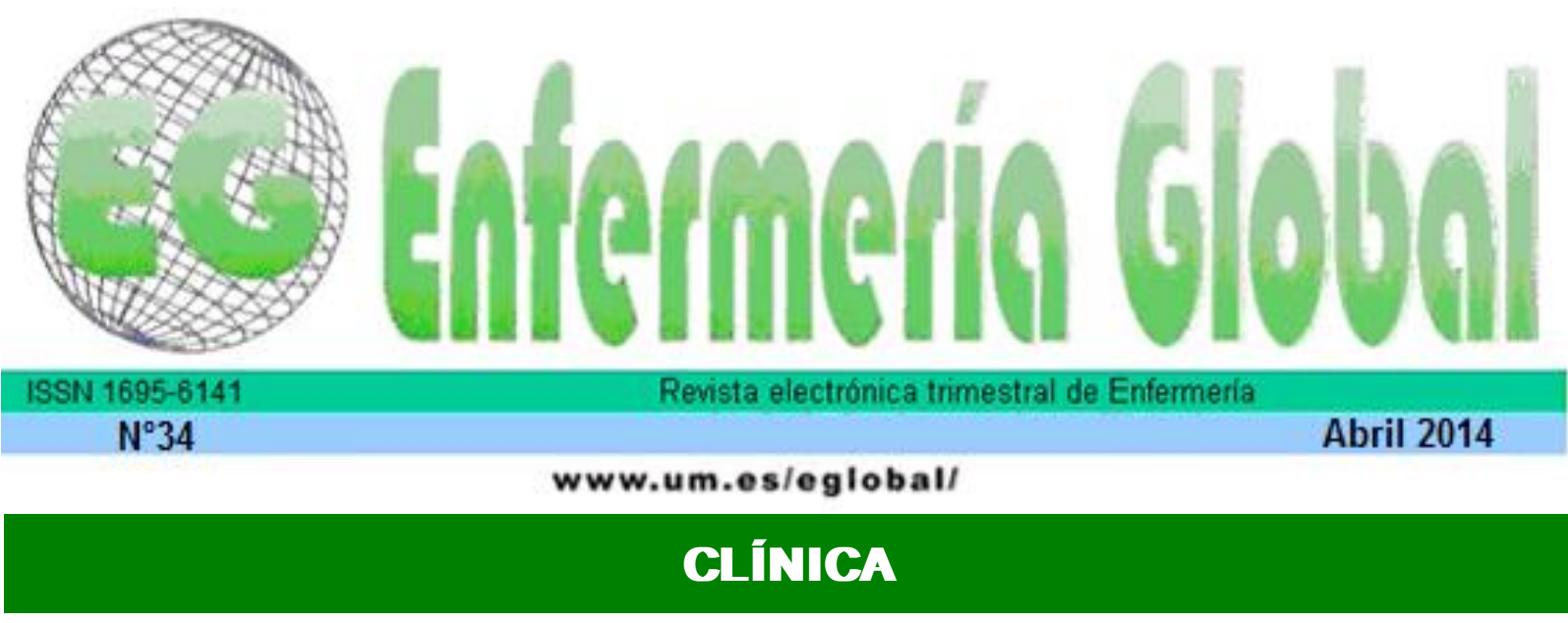

\title{
Condiciones de salud y funcionalidad de ancianos con Diabetes Mellitus tipo 2 en Atención Primaria
}

Condições de saúde e funcionalidade de idosos com Diabetes Mellitus tipo 2 na Atenção Primária à Saúde

Health conditions and functionality of the elderly with Diabetes Mellitus type 2 in Primary Health Care

\section{*Alves, Elaine Cristina Santos **Souza e Souza, Luís Paulo ** Santos Alves, Wellinson *Oliveira, Maricy Kariny Soares ${ }^{* * * *}$ Yoshitome, Aparecida Yoshie *****Gamba, Mônica Antar}

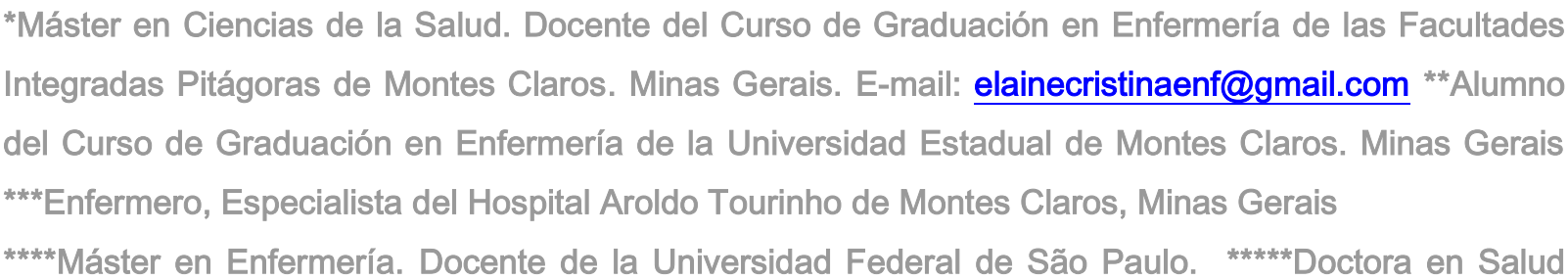
Pública. Docente de la Universidade Federal de São Paulo. Brasil.

Palabras clave: ancianos; actividades diarias; salud de la familia; condiciones de salud. Palavras chave: idosos; atividades cotidianas; saúde da família; condições de saúde. Keywords: ederly; everyday activities; family health; health conditions.

\section{RESUMEN}

Este estudio pretende identificar la salud y la funcionalidad de los ancianos con Diabetes Mellitus tipo 2 en la atención primaria de salud de Montes Claros-Minas Gerais, Brasil. Investigación cuantitativa y descriptiva, realizada en un área del centro de apoyo a la salud de la familia, 99 sujetos ancianos con Diabetes Mellitus tipo 2. La recolección de datos se llevó a cabo en los hogares de los encuestados, a través de cuestionario estructurado basado en herramientas de evaluación funcional y el Brasilian Multidimensional Functional Assesment Questionaire. Los datos se analizaron por Statistical Package for the Social Sciences. En relación con las condiciones clínicas, 94.9\% tienen comorbilidades; 54.5\% presentaron complicaciones de la diabetes; $84.8 \%$ informó sintiendo dolor en al menos una parte del cuerpo; $78 \%$ utiliza algún tipo de injerto/refuerzo; $26.3 \%$ y 22,2\% informó hospitalización y caída en el año pasado, respectivamente. 
Refiriéndose a las condiciones de funcionalidad, $59.6 \%$ de los encuestados reportó problemas geriátricos, $9.1 \%$ mencionó inmovilidad parcial; 30,3\% inestabilidad postural; $5.1 \%$ falla cognitiva; $28,3 \%$ incontinencia urinaria y $23.3 \%$ presenta dificultades en la realización de las actividades de la vida cotidiana. En cuanto al grado de dependencia, $79.8 \%$ presentó dependencia, siendo $40.4 \%$ suave; $20.2 \%$ moderada y $19,2 \%$ grave. Se concluye que el grado de dependencia de los ancianos encuestadas fue alto, requiriendo de la Atención Primaria de Salud responsable de la construcción y disponibilidad de las prácticas de salud apropiadas.

\section{RESUMO}

Este estudo objetivou identificar as condições de saúde e funcionalidade de idosos com Diabetes Mellitus Tipo 2 na Atenção Primária à Saúde de Montes Claros - Minas Gerais, Brasil. Pesquisa quantitativa e descritiva, realizada na área de abrangência de um Núcleo de Apoio à Saúde da Família, tendo como sujeitos 99 idosos com Diabetes Mellitus Tipo 2. A coleta dos dados foi realizada nos domicílios dos pesquisados, por meio de questionário estruturado baseado nos instrumentos de Avaliação Funcional e no Brasilian Multidimensional Functional Assesment Questionaire. Os dados foram analisados pelo Statistical Package for the Social Sciences. Em relação às condições clínicas, 94,9\% referiram co-morbidades; $54,5 \%$ apresentaram complicações do diabetes; $84,8 \%$ relataram sentir dor em pelo menos uma parte do corpo; 78\% utilizavam algum tipo de prótese/órtese; $26,3 \%$ e $22,2 \%$ informaram internação e queda no último ano, respectivamente.

Referente às condições de funcionalidade, $59,6 \%$ dos pesquisados relataram problemas geriátricos, sendo que $9,1 \%$ referiram imobilidade parcial; 30,3\% instabilidade postural; $5,1 \%$ insuficiência cognitiva; $28,3 \%$ incontinência urinária e $23,3 \%$ apresentaram dificuldades na realização das Atividades de Vida Diária. Quanto ao grau de dependência, 79,8\% apresentou dependência, sendo $40,4 \%$ leve; $20,2 \%$ moderada e $19,2 \%$ grave. Conclui-se que o grau de dependência dos idosos pesquisados foi alto, requerendo da Atenção Primária à Saúde responsável a construção e operacionalização de práticas de saúde adequadas.

\section{ABSTRACT}

This study aimed to identify the health and functionality of the elderly with Type 2 Diabetes Mellitus in primary health care of Montes Claros-Minas Gerais, Brazil, featuring clinical and functional conditions related to daily life activities. Quantitative and descriptive research held in a Core area of support to family health, having as subject 99 elderly subjects with Diabetes Mellitus type 2 . The data collection was conducted in the households of respondents, through structured questionnaire based on Functional assessment tools and the Brasilian Multidimensional Functional Assesment Questionaire. The data was analyzed by Statistical Package for the Social Sciences. In relation to clinical conditions, $94.9 \%$ have co-morbidities; $54.5 \%$ presented complications of diabetes; $84.8 \%$ reported feeling pain in at least one part of the body; $78 \%$ used some kind of prosthesis bracing; $26.3 \%$ and $22.2 \%$ reported hospitalization and fall last year, respectively.

Referring to the conditions of functionality, $59.6 \%$ of the surveyed reported geriatric problems, with partial immobility mentioned $9.1 \% ; 30.3 \%$ postural instability; $5.1 \%$ cognitive failure; $28.3 \%$ urinary incontinence and $23.3 \%$ presented difficulties in carrying out the activities of daily life. The degree of dependency, $79.8 \%$ presented dependency, being $40.4 \%$ mild; $20.2 \%$ moderate and $19.2 \%$. severe, It is concluded that the extent of tieing-in of seniors surveyed was high, requiring of primary health care in charge of the construction and operationalization of appropriate health practices that reduce vulnerabilities and maintain and protect the health of the elderly against early aggravations of comorbidities generated by Diabetes

\section{INTRODUCCIÓN}

El envejecimiento de la población es un fenómeno mundial, siendo una vivencia experimentada de diferentes formas por cada sociedad. Actualmente, existen 893 millones de personas con más de 60 años de edad en el mundo. En la primera mitad del presente siglo, esa cifra aumentará hasta alcanzar los 2,4 billones, la mayoría habitando en los países en desarrollo ${ }^{(1)}$. Según el Instituto Brasileño de Geografía y Estadística, en el 2010, la población brasileña era ya de 190.755.799 habitantes, de 
los cuales 20.590 .599 eran considerados ancianos (con edad de $\geq 60$ años), correspondientes al $10,8 \%$. $^{(2)}$.

Los avances médicos, farmacéuticos, sanitarios y tecnológicos, así como la disminución de las tasas de mortalidad y de fecundidad, han posibilitado el aumento de expectativa de vida de los ancianos, así como su contingente poblacional. Por otra parte, esa franja etária es más vulnerable a toda una serie de cambios físicos, fisiológicos y sicosociales que afectan a su calidad de vida. Por tanto, las discusiones sobre la salud deben volcarse siempre a los cuidados de los ancianos ${ }^{(3)}$.

El proceso de envejecimiento no se relaciona solo con las dolencias e incapacidades, aunque, estas afecciones son más frecuentes entre las personas con edad avanzada. En este sentido, se observa una tendencia creciente de personas de dicha edad con diagnóstico de enfermedades crónicas, presentando algún grado de dependencia funcional.

La Diabetes Mellitus (DM) tipo 2 se destaca entre estas, pues se observa un aumento notable en su prevalencia, siendo considerada una pandemia y un importante problema de Salud Pública. A pesar de la existencia de una diversificación cada vez mayor de la evolución tecnológica y farmacológica para el control de la DM el control efectivo exige un abordaje multifactorial, con la actuación y la participación de un equipo multiprofesional desde la prevención básica hasta la terciaria, evitando así la incapacidad funcional ${ }^{(4)}$.

La capacidad funcional es el estado de independencia para la realización de las actividades de la vida diaria que un ser humano debe tener para vivir con autonomía, tanto en su domicilio como en la comunidad. Los años de vida vividos asociados a las comorbilidades como la DM tipo 2 favorecen los chances de desarrollo de una dependencia funcional, como es destacado en varios estudios ${ }^{(3)}$.

A pesar de la amplitud del concepto, en la práctica se ha trabajado con el concepto de capacidad/incapacidad. La incapacidad funcional ha sido evaluada como la dificultad para realizar determinadas actividades de la vida cotidiana, en razón de alguna deficiencia. El estudio de la capacidad funcional ha ayudado a comprender cómo la longevidad contribuye a evaluar el estado de salud de los individuos diabéticos. Se sabe que la presencia de múltiples dolencias puede presentar diferentes grados de gravedad, influyendo en la vida diaria de las personas ${ }^{(4)}$.

Desde esa perspectiva, la capacidad funcional ha sido considerada un indicador del proceso salud-enfermedad. Estudios sobre los aspectos relacionados con el envejecimiento, las enfermedades crónicas y a la capacidad funcional son de gran importancia, pues ofrecerán informaciones relevantes sobre la calidad de vida de los ancianos y podrán influir significativamente en los cuidados a las personas ancianas con DM tipo $2^{(5)}$.

Considerando lo antes expuesto, este estudio pretendió identificar las condiciones de salud y funcionalidad de los ancianos con la Diabetes Mellitus Tipo 2 en la Atención Básica a la Salud, en la ciudad de Montes Claros, Minas Gerais, Brasil, caracterizando las condiciones clínicas y funcionales relacionadas a las Actividades de la Vida Diaria (AVD) en el domicilio. 


\section{METODOLOGÍA}

Este es un estudio cuantitativo, descriptivo y transversal, realizado en el área de alcance de un Núcleo de Apoyo a la Salud de la Familia (NASF) de la ciudad de Montes Claros, Minas Gerais, Brasil.

Los sujetos de la investigación fueron 99 ancianos con un diagnóstico médico de DM tipo 2 y cadastrados en el Programa HIPERDÍA. El HIPERDÍA es un Sistema de Cadastramiento y Acompañamiento de los Hipertensos y los Diabéticos captados en el Plano Nacional de Reorganización de la Atención a la hipertensión arterial y a la Diabetes Mellitus, en todas las unidades ambulatorias del Sistema Único de Salud, generando informaciones para los gerentes locales, gestores de las secretarías municipales, provinciales y el Ministerio de Salud.

La recolección de los datos fue realizada en el primer semestre de 2011 , en los domicilios de los ancianos, por medio de una encuesta estructurada basada en los instrumentos de evaluación funcional y la encuesta validada denominada Brasilian Multidimensional Functional Assesment Questionaire (BOMFAQ). Se trata de un cuestionario estructurado que proporciona los datos sociodemográficos, evalúa la percepción subjetiva del anciano, la salud física y mental (los aspectos cognitivos y emocionales), la independencia en el día a día, el soporte social y familiar así como la utilización de servicios ${ }^{(6)}$.

Las variables investigadas fueron las sociodemográficas y clínicas; aquellas relacionadas con los problemas geriátricos, el diagnóstico de la Diabetes Mellitus tipo 2 y la capacidad funcional.

Para el análisis de los datos, se utilizó el programa estadístico Statistical Package for the Social Sciences (SPSS) en su versión 18.0. Para el tratamiento estadístico, fueron realizados análisis descriptivos, generando las frecuencias y los porcentajes.

Resaltamos que todos los participantes firmaron un Término de Consentimiento Libre y Consciente, manteniendo así el sigilo sobre las informaciones recolectadas. El presente estudio fue aprobado por el Comité de Ética en Pesquisa (CEP) de la Universidad Federal de São Paulo, con parecer número 0276/2010.

\section{RESULTADOS}

Con relación a los datos sociodemográficos, se percibió que el 73,7\% ( $n=72)$ eran del sexo femenino; presentaban una edad promedio de 68,8 años (variación patrón de 7,2 años y promedio de 67), con una oscilación entre un mínimo de 56 años y el máximo de 91 años; el 97\% ( $n=96)$ tenía grado de escolaridad bajo; el 45,5\% ( $n=45)$ eran viudos; el $44,4 \%(n=44)$ vivía con sus hijos; el $90,9 \%(n=90)$ no practicaba ninguna actividad remunerada; y el $57,6 \%(n=57)$ poseía uma renta mensual de un salario mínimo, conforme evidencia la tabla 1. 
Tabla 1 - Distribución de los ancianos con diabetes mellitus según las variables sociodemográficas. Montes Claros, Minas Gerais, 2011.

\begin{tabular}{|c|c|c|c|}
\hline Variables & & $\mathbf{n}$ & $\%$ \\
\hline \multirow{3}{*}{ Sexo } & Masculino & 27 & 27,3 \\
\hline & Femenino & 72 & 72,7 \\
\hline & Hasta 64 años & 38 & 38,4 \\
\hline \multirow[t]{3}{*}{ Edad } & 64 a 71 años & 28 & 28,3 \\
\hline & 72 o más & 33 & 33,3 \\
\hline & Analfabeto & 41 & 41,5 \\
\hline \multirow[t]{7}{*}{ Escolaridad } & Sabe leer/escribir & 34 & 34,3 \\
\hline & Primaria completa & 21 & 21,2 \\
\hline & Gimnasio completo & 01 & 1,0 \\
\hline & Enseñanza media completa & 02 & 2,0 \\
\hline & Enseñanza superior completa & - & \\
\hline & Soltero & 05 & 5,1 \\
\hline & Casado & 44 & 44,3 \\
\hline \multirow[t]{5}{*}{ Estado civil } & Separado/divorciado & 05 & 5,1 \\
\hline & Viudo & 45 & 45,5 \\
\hline & Unión estable & - & \\
\hline & Solo & 14 & 14,1 \\
\hline & Sólamente con cuidador profesional & 01 & 1,0 \\
\hline \multirow[t]{5}{*}{ Con quién vive } & Con cónjuge & 24 & 24,2 \\
\hline & Con otros de su generación & 05 & 5,1 \\
\hline & Con hijos & 44 & 44,4 \\
\hline & Con nietos & 08 & 8,1 \\
\hline & Otros & 03 & 3,1 \\
\hline & No & 90 & 90,9 \\
\hline \multirow[t]{3}{*}{ remunerada } & Sí & 09 & 9,1 \\
\hline & Sin renta & 02 & 2,0 \\
\hline & Hasta 1 salario mínimo & 57 & 57,6 \\
\hline \multirow{4}{*}{ Renta mensual } & De 2 a 3 salarios mínimos & 36 & 36,4 \\
\hline & De 3 a 4 salarios mínimos & 03 & 3,0 \\
\hline & De 4 a 5 salarios mínimos & 01 & 1,0 \\
\hline & Más de 5 salarios mínimos & - & \\
\hline Total & & 99 & 100,0 \\
\hline
\end{tabular}

Fuente: Investigación de campo, 2011

Con relación a las variables clínicas, los resultados fueron agrupados en la tabla 2.

Tabla 2 - Distribución de los ancianos con diabetes mellitus según las variables clínicas. Montes Claros, Minas Gerais, 2011.

\begin{tabular}{llll}
\hline Variables & & N & $\%$ \\
\hline Siente dolor & No & 15 & 15,2 \\
& Sí & 84 & 84,8 \\
Intensidad del dolor & Leve & 16 & 16,2 \\
& Moderado & 35 & 35,4 \\
& Severo & 31 & 31,3 \\
Uso de prótesis & No respondieron & 17 & 17,1 \\
Prótesis ocular & No & 21 & 21,2 \\
& Sí & 78 & 78,8 \\
& No & 74 & 74,7
\end{tabular}




\begin{tabular}{|c|c|c|c|}
\hline \multirow{3}{*}{ Prótesis auditiva } & Sí & 25 & 25,3 \\
\hline & No & 99 & 100,0 \\
\hline & Sí & - & - \\
\hline \multirow{2}{*}{ Prótesis ortopédica } & No & 96 & 97,0 \\
\hline & Sí & 03 & 3,0 \\
\hline \multirow{2}{*}{ Otras } & No & 28 & 28,3 \\
\hline & Sí & 71 & 71,7 \\
\hline \multirow[b]{2}{*}{ Uso de medicamentos prescritos } & No & 01 & 1,0 \\
\hline & Sí & 98 & 99,0 \\
\hline \multirow[t]{3}{*}{ Uso de Automedicación } & No & 58 & 58,6 \\
\hline & Sí & 38 & 38,4 \\
\hline & No respondieron & 03 & 3,0 \\
\hline \multirow{2}{*}{ Internación en el último año } & No & 73 & 73,7 \\
\hline & Sí & 26 & 26,3 \\
\hline \multirow{2}{*}{ Caída en el último año } & No & 77 & 77,8 \\
\hline & Sí & 22 & 22,2 \\
\hline \multirow{2}{*}{ Una caída } & No & 92 & 92,9 \\
\hline & Sí & 7 & 7,1 \\
\hline \multirow{2}{*}{ Entre dos y cuatro } & No & 89 & 89,9 \\
\hline & Sí & 10 & 10,1 \\
\hline \multirow{4}{*}{ Cinco o más } & No & 94 & 94,9 \\
\hline & Sí & 05 & 5,1 \\
\hline & Tropezón & 14 & 14,1 \\
\hline & Pérdida de la consciencia & 04 & 4,0 \\
\hline \multirow{5}{*}{ Causas de la caída } & Sin motivo & 01 & 1,0 \\
\hline & Dolencia aguda & - & - \\
\hline & Otras & 05 & 5,1 \\
\hline & No respondieron & 75 & 75,8 \\
\hline & No & 18 & 18,2 \\
\hline \multirow[t]{2}{*}{ Fracturas } & Sí & 04 & 4,0 \\
\hline & No respondieron & 77 & 77,8 \\
\hline \multirow{3}{*}{$\begin{array}{l}\text { Influencia de la caída en las } \\
\text { actividades cotidianas }\end{array}$} & No & 14 & 14,1 \\
\hline & Sí & 06 & 6,1 \\
\hline & No respondieron & 79 & 79,8 \\
\hline \multicolumn{2}{|l|}{ Total } & 99 & 100,0 \\
\hline
\end{tabular}

Fuente: Pesquisa de campo, 2011.

Se verificó que el $84,8 \%(n=84)$ sentía dolor, siendo un $35,4 \%(n=35)$ con intensidad moderada; el $78,8 \%(n=78)$ hacían uso de algún tipo de prótesis, siendo que la variable "otros tipos de prótesis" fue la más citada, totalizando un $71,7 \%(n=71)$, seguida por las prótesis oculares, con el 25,3\% ( $n=25)$. La mayoría de los participantes, el $99 \%(n=98)$, hacía uso de medicamentos prescritos; el $58,6 \%(n=58)$ no realizan automedicación, mientras el 73,7\% $(n=73)$ no sufrió ingreso en el último año.

Con relación a la presencia de caídas en el último año, el $77,8 \% \quad(n=77)$ no presentaron ese cuadro, siendo que de los $22(22,2 \%)$ ancianos que presentaron, el $10,1 \%(n=10)$ tuvieron entre dos y cuatro caídas, siendo la principal causa el tropezón, cuantificando un $14,1 \%(n=14)$. La mayoría no presentó fractura, el $(18,2 \%, n=18)$ y un $79,8 \% \quad(n=79)$ no respondieron si las caídas influyeron en sus actividades cotidianas. 
Otro aspecto analizado en el presente estudio fueron las variables relacionadas con los problemas geriátricos, conforme muestra la tabla 3 .

Tabla 3 - Distribución de los ancianos con diabetes mellitus según las variables relacionadas a los problemas geriátricos. Montes Claros, Minas Gerais, 2011

\begin{tabular}{llll}
\hline Variables & & n & $\%$ \\
\hline Problemas geriátricos & No & 40 & 40,4 \\
& Sí & 59 & 59,6 \\
Inmovilidad parcial o total & No & 90 & 90,9 \\
Inestabilidad de postura & No & 09 & 9,1 \\
& Sí & 69 & 69,7 \\
latrogenia & No & 30 & 30,3 \\
& Sí & 82 & 82,8 \\
Incontinencia urinaria & No & 17 & 17,2 \\
& Sí & 71 & 71,7 \\
Incontinencia fecal & No & 28 & 28,3 \\
& Sí & 98 & 99,0 \\
Insuficiencia cognitiva & No & 01 & 1,0 \\
Insuficiencia familiar & Sí & 94 & 94,9 \\
& No & 05 & 5,1 \\
\multirow{4}{*}{ Memoria } & Sí & 88 & 88,9 \\
& Excelente & 11 & 11,1 \\
& Buena & 07 & 7,1 \\
& Regular & 48 & 48,5 \\
Percepción de salud & Pésima & 37 & 37,4 \\
& No respondieron & 06 & 6,0 \\
& Optima & 09 & 1,0 \\
& Buena & 65 & 9,1 \\
Total & Mala & 18 & 65,7 \\
Salud comparada & Pésima & 07 & 18,2 \\
& Mucho peor & 02 & 7,0 \\
& Peor & 2,0 \\
& Igual & 08 & 8,0 \\
& Mejor & 27 & 27,3 \\
& Mucho mejor & 45 & 45,5 \\
& No respondieron & 15 & 15,2 \\
& & 02 & 2,0 \\
& 99 & 100,0 \\
\hline
\end{tabular}

Fuente: Investigación de campo, 2011.

Se percibe que el $59,6 \%(n=59)$ de los entrevistados relataron tener problemas geriátricos. De ellos un $30,3 \%(n=30)$ relataron inestabilidad de postura y un $28,3 \%$ $(\mathrm{n}=28)$ de incontinencia urinaria. La memoria autoreferida de los entrevistados fue considerada "buena", totalizando un $48,5 \%(n=48)$, seguida de "regular", que cuantificó un $37,4 \%(n=37)$. Al ser preguntados sobre la percepción con relación a la salud, un $65,7 \%$ ( $n=65)$ clasificaron como "buena" y, comparándola con la salud de otros ancianos portadores de DM tipo 2, 45,4\% ( $n=45)$ clasificaron que su salud se encontraba mejor.

Por otra parte, la tabla 4 describe a los pacientes según el diagnóstico de Diabetes Mellitus tipo 2. Se notó que el $24,2 \%(n=24)$ de los ancianos relataron poseer más de 20 años de diagnóstico; un 94,9\% $(n=94)$ afirmaron presentar otros problemas 
además de la DM, siendo que el $88,9 \% \quad(n=88)$ también son portadores de enfermedades cardiovasculares. Con relación a las complicaciones generadas por la DM, el $54,5 \%(n=54)$ afirmó haber desarrollado algún problema, siendo la retinopatía diabética (RD) el mayor número de casos, habiéndose encontrado un $38,4 \%(n=38)$. Con relación al tratamiento, el $98 \%(n=97)$ realizaban tratamiento y un $70,3 \%(n=70)$ dijeron que la DM tipo 2 no trajo dificultades para realizar sus actividades diarias.

Con respecto a las orientaciones realizadas por la Estrategia Salud de la Familia (ESF) o NASF, el 89,9\% ( $n=89)$ dijeron haber recibido algún tipo de orientación.

Tabla 4 - Distribución de los pacientes con diabetes mellitus según las variables relacionadas al diagnóstico de DM. Montes Claros, Minas Gerais, 2011.

\begin{tabular}{|c|c|c|c|}
\hline \multirow[t]{2}{*}{ Variables } & & $\mathbf{n}$ & $\%$ \\
\hline & Menos de 1 año & 01 & 1,0 \\
\hline \multirow{7}{*}{ Tiempo de diagnóstico } & 1 a 3 años & 05 & 5,1 \\
\hline & 4 a 7 años & 21 & 21,2 \\
\hline & 8 a 11 años & 18 & 18,2 \\
\hline & 12 a 15 años & 20 & 20,2 \\
\hline & 16 a 19 años & 09 & 9,1 \\
\hline & 20 o más & 24 & 24,2 \\
\hline & No respondieron & 01 & 1,0 \\
\hline \multirow{2}{*}{$\begin{array}{l}\text { Problema de salud, además de la } \\
\text { diabetes }\end{array}$} & No & 05 & 5,1 \\
\hline & Sí & 94 & 94,9 \\
\hline \multirow{2}{*}{ Depresión } & No & 93 & 93,9 \\
\hline & Sí & 06 & 6,1 \\
\hline \multirow{2}{*}{ Dolencia neurosiquiátrica } & No & 99 & 100,0 \\
\hline & Sí & - & - \\
\hline \multirow{2}{*}{ Neoplasia } & No & 98 & 99,0 \\
\hline & Sí & 01 & 1,0 \\
\hline \multirow{2}{*}{ Dolencia cardiovascular } & No & 11 & 11,1 \\
\hline & Sí & 88 & 88,9 \\
\hline \multirow{3}{*}{ Dolencia pulmonar } & No & 98 & 99,0 \\
\hline & Sí & 01 & 1,0 \\
\hline & No & 91 & 91,9 \\
\hline Dolencia gastrointestinal & Sí & 08 & 8,1 \\
\hline \multirow[t]{2}{*}{ Otras } & No & 79 & 79,8 \\
\hline & Sí & 20 & 20,2 \\
\hline \multirow[t]{3}{*}{ Complicaciones de la diabetes } & No & 44 & 44,5 \\
\hline & Sí & 54 & 54,5 \\
\hline & No respondieron & 01 & 1,0 \\
\hline \multirow[t]{3}{*}{ Retinopatía } & No & 60 & 60,6 \\
\hline & Sí & 38 & 38,4 \\
\hline & No respondieron & 01 & 1,0 \\
\hline \multirow[t]{2}{*}{ Dolencia arterial coronaria } & No & 86 & 86,9 \\
\hline & Sí & 12 & 12,1 \\
\hline
\end{tabular}




\begin{tabular}{|c|c|c|c|}
\hline \multicolumn{2}{|l|}{ Variables } & $n$ & $\%$ \\
\hline & No & 87 & 87,9 \\
\hline \multirow[t]{2}{*}{ Nefropatía } & Sí & 11 & 11,1 \\
\hline & No respondieron & 01 & 1,0 \\
\hline \multirow[t]{3}{*}{ Dolencia cerebrovascular } & No & 94 & 94,9 \\
\hline & Sí & 04 & 4,0 \\
\hline & No respondieron & 01 & 1,0 \\
\hline \multirow[t]{3}{*}{ Neuropatía } & No & 91 & 91,9 \\
\hline & Sí & 07 & 7,1 \\
\hline & No respondieron & 01 & 1,0 \\
\hline \multirow[t]{3}{*}{ Dolencia vascular perisférica } & No & 90 & 90,9 \\
\hline & Sí & 08 & 8,1 \\
\hline & No respondieron & 01 & 1,0 \\
\hline \multirow[t]{2}{*}{ Orientación ESF } & No & 10 & 10,1 \\
\hline & Sí & 89 & 89,9 \\
\hline \multirow[t]{2}{*}{ Tratamiento para la diabetes } & No & 02 & 2,0 \\
\hline & Sí & 97 & 98,0 \\
\hline \multirow{2}{*}{$\begin{array}{l}\text { La Diabetes dificulta la } \\
\text { realización de las Actividades }\end{array}$} & No & 70 & 70,7 \\
\hline & Sí & 29 & 29,3 \\
\hline Total & & 99 & 100,0 \\
\hline
\end{tabular}

Fuente: Pesquisa de campo, 2011.

Para la encuesta de evaluación de la funcionalidad de las Actividades de la Vida Diaria (AVD), se utilizó el BOMFAQ, siendo que el cálculo del promedio fue obtenido por medio de la suma de las respuestas dadas por los encuestados en cada una de las preguntas relacionadas a las Actividades Básicas e Instrumentales de la Vida Diaria, de acuerdo a la tabla 5.

Tabla 5 - Distribución de los ancianos con diabetes mellitus según el nivel de dependencia basado en la evaluación BOMFAQ. Montes Claros, Minas Gerais, 2011.

\begin{tabular}{lll}
\hline Nivel de dependencia & $\mathbf{n}$ & $\%$ \\
\hline Sin dependencia & 20 & 20,2 \\
Dependencia leve & 40 & 40,4 \\
Dependencia moderada & 20 & 20,2 \\
Dependencia grave & 19 & 19,2 \\
Total & $\mathbf{9 9}$ & $\mathbf{1 0 0 , 0}$ \\
\hline
\end{tabular}

Fuente: Pesquisa de campo, 2011.

La puntuación promedio fue de 7,1 puntos, con una oscilación patrón de 8,2 puntos, de un valor mínimo de 0 puntos y un máximo de 15. El grado de dependencia fue calculado considerándose los siguientes parámetros de corte para la puntuación: $0=$ sin dependencia; de 1 a 3 = dependencia leve; de 4 a 6 = dependencia moderada; 7 o más $=$ dependencia grave.

Se nota que la mayoría de los ancianos investigados presentó algún nivel de dependencia, totalizando un $79,8 \%(n=79)$. 


\section{DISCUSIÓN}

En el presente estudio, se percibe que la mayor parte de los ancianos era del sexo femenino; con edad promedio de 68,8 años; con baja escolaridad; viudos; residían con sus hijos o cónyuges y poseían una renta mensual de solo un salario mínimo. Corroborando este estudio, resultados semejantes fueron encontrados por investigadores en una Estrategia de Salud de la Familia en Goiânia. ${ }^{(7)}$.

Se resalta la importancia del aumento simultáneo de la expectativa de vida y de la prevalencia de DM en los ancianos. Datos del Ministerio de Salud apuntan un aumento de la prevalencia de la DM tipo 2 con la elevación de la franja etária, con índices del $17 \%$ entre los mayores de sesenta años ${ }^{(8)}$.

En ese contexto, en un estudio realizado con las características de los asociados del plano de salud de la Cooperativa de los Usuarios de los Servicios de Salud del Valle del Rio de las Campanas ${ }^{(9)}$, se constató una relación directa entre la prevalencia de la incapacidad funcional y la edad, o sea, a medida que los grupos etários se elevaban, aumentaban las prevalencias del desenlace, existiendo una tendencia lineal significativa $(p<0,01)$. Los resultados mostraron que los individuos, a partir de los 80 años, presentaron una mayor probabilidad de incapacidad funcional. Lo mismo ocurrió en otro estudio, donde los investigadores consiguieron hallar una asociación estadística significativa para las variables: sexo femenino y edad por encima de 70 años relacionadas al chance de desarrollar una dependencia para las Actividades de la Vida Diaria (AVD) ${ }^{(10)}$.

Se observó que con relación a la escolaridad, el 41,5\% de los entrevistados relataron ser analfabetos, dato ese preocupante cuando se trata de la obtención de informaciones nuevas y de alteraciones cognitivas, lo que dificulta el aprendizaje de las acciones educativas de salud desarrolladas por la ESF. El análisis del grado de instrucción es de suma importancia, toda vez que su condición de baja escolaridad puede impedir el acceso a las informaciones, ofreciendo menores oportunidades de acceso a las acciones para el autocuidado de la salud ${ }^{(11)}$.

Al comparar el nivel educacional entre los ancianos diabéticos y no diabéticos con las mismas características sociodemográficas, investigadores evidenciaron que el nivel educativo de los individuos diabéticos es relativamente más bajo. Ese dato merece ser destacado, ya que los ancianos con nivel más bajo de escolaridad tienen una mayor probabilidad de presentar dependencia física y mayores características de cuadros demenciales ${ }^{(9)}$.

Algunos autores han evidenciado una fuerte asociación entre el control glucémico y las alteraciones cognitivas, sugiriendo que la hiperglucemia crónica puede contribuir al desarrollo de deficiencias orgánicas en el cerebro, manifestadas como una reducción total de la eficiencia mental, y en personas con diabetes mellitus tipo 2 puede perjudicar significativamente la velocidad del procesamiento de las informaciones ${ }^{(12-}$ 13).

Así, la baja escolaridad asociada a la presencia del déficit cognitivo se convierte en un gran obstáculo a ser vencido por los ancianos con DM para mantener la adhesión a las prácticas adecuadas y seguras en la autoaplicación de la insulina. De ese modo, al proponer las intervenciones de salud, los profesionales en la Atención Básica de 
Salud deben considerar que las limitaciones funcionales y de aprendizaje pueden perjudicar el desarrollo de las habilidades para el autocuidado.

Esta preocupación puede ser confirmada en un estudio desarrollado en la región del Triángulo Mineiro, Minas Gerais, donde se observó que cuanto menores fueron los años de estudio, mayores fueron los chances para los usuarios en autoaplicarse la insulina correctamente. Esta dificultad se extiende también a los otros procedimientos de autocontrol de la DM como la auto medición de la glucemia, el uso de los medicamentos y los cuidados de los pies que exijan capacidad de comprensión de informaciones nuevas. Por estas razones, los profesionales de la salud de la Estrategia Salud de la Familia deberán hacer intervenciones sustentadas en las capacidades funcionales y educación promoviendo los procesos de adaptación de los ancianos con DM, pero así que sean detectadas las dificultades para lograr el objetivo de estimular la autoaplicación de la insulina, el profesional de la salud deberá involucrar a la familia en la responsabilidad de este procedimiento ${ }^{(14)}$.

Sobre las cuestiones de vivienda, se observó que la mayoría de los entrevistados vive con familiares, como el cónyuge o sus hijos. Este aspecto puede ser positivo para la manutención de la funcionalidad, que debe ser explotado por el equipo de ESF, junto a la familia y en la promoción de la educación en salud, convirtiéndose en una aliada para el desarrollo de la planificación con relación a los cuidados y medidas de control con la enfermedad. También hay que considerar que una pequeña parte de los entrevistados, el $14 \%(n=14)$, vive solo, situación que significa una mayor fragilidad para los mismos. Los estudios afirman que el anciano con DM necesita del apoyo y desarrolla algún grado de dependencia para la realización de las $A V D^{(10)}$.

Con relación a la renta, algunos autores afirman que una baja renta representa un factor capaz de comprometer las condiciones de salud de los ancianos, de un modo general, en especial entre los portadores de $\mathrm{DM}^{(15)}$. La adhesión al tratamiento médico y dietético necesita del apoyo presupuestario, toda vez que en la actual investigación, el $98,2 \%$ de los ancianos estudiados hacían uso regular de medicamentos y algunas veces, necesitan gastar en insumos, alimentación y medicinas que no consiguen en la red básica, comprometiendo por lo menos el $10 \%$ de sus recursos financieros para lograr un control clínico sostenido adecuado. Esta situación es aún peor para aquellos que no poseen rendimientos financieros propios ${ }^{(15)}$.

Estudios brasileños utilizando datos de la Pesquisa Nacional por Muestreo de Domicilios (PNAD) ha mostrado que la capacidad funcional de los ancianos está fuertemente influida por la renta domiciliaria per cápita. Esa asociación persiste a pesar de los ajustes posteriores por varios indicadores de las condiciones de salud ${ }^{(16)}$. En otro estudio desarrollado en Ubá, Minas Gerais, los autores constataron que los ancianos del sexo femenino, viudas, analfabetas, que viven solas, con baja renta, sedentarias e inactivas en el mercado de trabajo, presentaron mayores chances para el comprometimento de la capacidad funcional ${ }^{(17)}$.

En cuanto a las características clínicas de los estudiados, se observó que los resultados encontrados concuerdan con otros estudios, principalmente relacionadas con el dolor, al referir que la mayoría de esos pacientes presentaron dolor relacionado a la condición clínica ${ }^{(18)}$.

El dolor es definido como un síntoma subjetivo de gran importancia clínica, y cuando está relacionado con la Neuropatía Diabética (ND), puede influir de modo significante 
en la calidad de vida del individuo(19). En este sentido, es necesario que los profesionales de la ESF estén atentos a los síntomas de dolor y la Neuropatía Diabética en los participantes del estudio, ya que el $84,8 \%$ de los entrevistados relataron sentir dolor, siendo que el $35,6 \%$ lo clasificaron como moderado, lo que puede ser un factor influyente en las dificultades para realizar las AVD.

Con relación al uso de los medicamentos se destacó la prevalencia del uso de medicamentos en la población estudiada de un 72,1\%. La mayor convivencia con problemas crónicos de salud convierte a los ancianos en grandes consumidores de servicios de salud y de medicamentos ${ }^{(20)}$.

En cuanto a la relación de los indicadores de salud, el relato de caídas, el uso de medicamentos, la presencia de condiciones crónicas de salud autoreferida mostraron una asociación estadística significante con el compromiso de la capacidad funcional. La población anciana registra una fragilización y una probabilidad de complicaciones mayores, siendo la que proporcionalmente más utiliza los servicios de salud y medicamentos. Sin embargo, es necesaria una mayor atención ante la cultura de la medicación, especialmente entre los ancianos ya que las reacciones adversas a los medicamentos son mayores en ese grupo ${ }^{(17)}$.

El hecho de que un $22,2 \%$ de los entrevistados refirieron haber sufrido entre dos y cuatro $(10,1 \%)$ caídas durante el último año y entre las causas más comunes los tropezones $(14,1 \%)$, es algo preocupante para la evaluación de la capacidad funcional, siendo todavía más relevante por tratarse de ancianos con la DM tipo 2, imponiéndose como un gran riesgo la salud e independencia de los mismos, pues puede estar asociado a las complicaciones de dolencias referidas por un $54,5 \%$ de estos. Confirmando los resultados de la presente investigación, un estudio reveló que la caída estuvo estadísticamente asociada a la dependencia en las $A V D^{(7)}$. En ese sentido, para prevenir esos episodios, se aconseja retirar tapetes, objetos que dificulten la circulación y conlleven al tropiezo, sustituyéndolos por piso adherente, iluminación adecuada en todos los ambientes y barreras arquitectónicas que faciliten la locomoción del anciano, como presencia de pasamanos en las escaleras o pasillos, ausencia de pisos cuarteados o desnivelados y las escaleras no pueden tener una altura que conlleve a un esfuerzo exagerado para usarlas ${ }^{(7)}$.

Los investigadores certifican que el relato de caídas en los últimos tres meses se mostró como un indicador de peor capacidad funcional, siendo más frecuente entre las mujeres. Las caídas, que están presentes en cerca del $70 \%$ de los accidentes con los ancianos pueden representar una pérdida significativa de la capacidad funcional. Ante los datos citados, se convierten en algo imperativo las acciones de la ESF en el seguimiento de los ancianos que sufrieron caídas, así como una evaluación ambiental durante las visitas domiciliarias para el manejo de las adaptaciones del ambiente doméstico con el objetivo de reducir los riesgos de caídas, ya que estos pueden tener menor sensibilidad plantar y de reflejos propioceptivos ${ }^{(7)}$.

Con relación a los problemas de la población anciana, es común observar manifestaciones atípicas en función de su alta frecuencia, siendo denominadas "los gigantes de la geriatría", las cuales son: inestabilidad de postura; incontinencias; insuficiencia cerebral, e inmovilidad e iatrogenia ${ }^{(14)}$. En el presente estudio, la mayoría (el 59,6\%) tenía algún problema geriátrico, siendo la inestabilidad de postura (un $30,3 \%$ ) y la incontinencia urinaria ( un $8,3 \%$ ) los más citados. 
Envejecer forma parte del proceso fisiológico y dinámico, en el cual ocurre una cascada de modificaciones en la capacidad de adaptación homeostática, suprimiendo así algunas de las etapas del control de la postura. Eso lleva al aumento de la inestabilidad de la postura del individuo y cuando es asociado a la DM, conduce al individuo, de forma progresiva, a perjuicios aún mayores ${ }^{(13,21)}$.

Es sabido que los ancianos, generalmente, tienen un esquema médico polifármaco, provocando que se produzca una interacción entre esos medicamentos, causando como efecto colateral la inestabilidad de postura. Determinados autores revelan que las disfunciones motoras, sensoriales, la depresión, el déficit cognitivo y de medicamentos (los benzodiazepínicos, antisicóticos, antidepresivos y otros) ocasionan la inestabilidad de postura y la alteración del paso, aumentando las posibilidades de riesgos para las caídas, que podrán perjudicar la ejecución de las actividades cotidianas ${ }^{(13)}$. Eso puede ser confirmado en este estudio, donde hubo un relato de la prevalencia de inestabilidad de postura, revelando un tanto por ciento importante de ocurrencia de caídas en los entrevistados desvelando la asociación con dependencia para las AVD.

Con respecto a la incontinencia urinaria, algunas autoras (22) explican que la asociación entre la DM y la incontinencia urinaria ocasiona un aumento de la vulnerabilidad del assoalho pélvico, debido a un cambio del tejido biológico y a los nervios del músculo pélvico o consecuencia de lesiones en las enervaciones neuropáticas autónomas de la vejiga o por el aumento de la frecuencia urinaria causada por la hiperglucemía provocada por el aumento del volumen urinario. Además de los problemas de convivencia social ${ }^{(22)}$, este cuadro acarrea problemas clínicos como interrupción del sueño y predisposición a las caídas durante la noche, por el el aumento de veces que necesita ir al baño.

Resulta relevante discutir que a pesar de que la mayoría de los ancianos encuestados no presenta incontinencia urinaria, 28 de los estudiados respondieron positivamente a esta pregunta, exigiendo mayor atención por los profesionales de la ESF, principalmente el enfermero durante las consultas de enfermería, por medio de la aplicación de la puntuación para la evaluación de este cuadro dentro de las acciones de la Unidad.

Con relación a la insuficiencia cognitiva, el 94,9\% $(n=94)$ de los entrevistados con la DM tipo 2 no presentaron. Sin embargo, algunos estudios sugieren que los ancianos con DM tipo 2 son más propensos a presentar algún déficit cognitivo si los comparamos con aquellos sin la enfermedad. Algunas evidencias sugieren que los déficits del aprendizaje y de la memoria en esos individuos pueden ser el resultado de una interacción sinérgica entre las alteraciones del metabolismo relacionadas con la diabetes, en que los cambios en la concentración de la glucose sanguínea afectan rápidamente a la función cerebral, y los cambios estructurales y funcionales que ocurren en el sistema nervioso central, provocados por el proceso normal del envejecimiento ${ }^{(23)}$.

La memoria autorreferida de los entrevistados fue considerada buena (el 48,5\%) a regular (el $37,4 \%$ ), sin embargo otros investigadores ${ }^{24}$ describen una asociación significativa con la co-ocurrencia de um bajo desempeño cognitivo con otras dolencias, condiciones crónicas y con incapacidad de ejecutar tareas específicas. En la muestra de ancianos encuestados por los estudiosos citados, más de la mitad presentó entre un 20 y un $40 \%$ de dificultad funcional especificamente asociada con el 
desempeño cognitivo bajo. No obstante, el bajo desempeño cognitivo no fue uniformemente asociado a limitaciones en las AVD ${ }^{(24)}$.

Con relación a la autopercepción de la salud, muchos entrevistados consideran su salud buena (el 65,7\%), y cuando se compara con otras personas con DM tipo 2, el $45,5 \%$ relataron estar mejor. Este resultado se contrapuso a los resultados de otro estudio realizado en la Región Metropolitana de Belo Horizonte, Minas Gerais, donde los análisis multivariados de los factores asociados a la incapacidad moderada obtuvieron asociaciones positivas e independientes para edades iguales o superiores a los 80 años (OR = 3,77; IC95\%: 2,14-6,66), peor percepción de la salud (OR =1,92; IC95\%: 1,13-3,26 para regular y OR $=5,27$; IC95\%:2,89-9,63 para mala o muy mala $)^{(10)}$. En ese sentido, la autoevaluación del estado de salud es variable previsora de la incapacidad funcional y refleja una percepción íntegra del individuo, que incluye las dimensiones biológica, social y psicosocial ${ }^{(17)}$.

Sobre el tiempo del diagnóstico de la DM, el estudio verificó que la mayoría de la población investigada tenía más de 20 años y presentan complicaciones generadas por la DM, siendo la retinopatía el mayor número de casos. En cuanto a las variables relacionadas al tiempo de diagnóstico de la DM, algunos autores relatan que la mayor ocurrencia de los problemas de salud referidos estaba entre los sujetos con un plazo de diagnóstico entre los 5 y los 10 años y 20 años o más. De esta forma, es necesario implementar acciones de salud buscando la exposición a los factores de riesgo, el diagnóstico precoz y la protección de las complicaciones en los diversos niveles de atención a la salud ${ }^{(25)}$.

En cuanto a la presencia de otras comorbidades, el $88,9 \%$ de los entrevistados refirieron la dolencia cardiovascular. Algunos estudios demuestran que entre las enfermedades asociadas a la DM tipo 2, la mayor es la HAS y que esta condición agrava todavía más el cuadro, posibilitando mayores chances de esos pacientes a manifestar complicaciones. Investigadores evidenciaron en estudios realizados que la HAS fue la dolencia más observada en los pacientes con DM del tipo 2, y cuando aparecen asociadas, constituyen los principales factores de riesgo para las dolencias cardiovasculares siendo la principal causa de óbito en Brasil. ${ }^{25}$ Otro estudio ${ }^{9}$ mostró una asociación estadística con relación a la presencia de las comorbidades, o sea, cuanto más elevada es la presencia de esas comorbidades, mayor es la prevalencia de desarrollar incapacidades funcionales. Una de las estrategias para mitigar y acompañar estos riesgos es el monitoramiento eficaz de estos clientes a través del programa HIPERDIA ya implementado en los equipos de Salud de la Familia.

Los diabéticos que relataron tener una o dos dolencias crónicas asociadas, cifra que equivale a casi dos veces más al compararse con los no diabéticos, pueden desarrollar mayores dificultades en la realización de las AVD, siendo un factor importante relacionado con la pérdida de la funcionalidad de los ancianos ${ }^{(26)}$.

Además de las complicaciones cardiovasculares, los pacientes con DM pueden presentar problemas de visión como la Retinopatía Diabética (RD), la Nefropatía Diabética (ND) y la Neuropatía sensitiva distal. Se verificó también que el 54,5\% poseían complicaciones generadas por la DM, habiendo presentado un $38,5 \%$ la RD y un $11,1 \%$ las nefropatías. Corroborando estos resultados, algunos estudios revelaron que cuando la DM está asociada a la deficiencia visual no suceptible de corrección y alteración de la agudeza visual, predispone a un mayor chance de desarrollar dependencia para las $\operatorname{AVD}^{(7,27-28)}$. 
El seguimiento de la RD y de la ND de forma más sistemática, por medio de la adopción de estas intervenciones, podría ayudar en la prevención de las complicaciones que la mayor parte de las veces son irreversibles en la DM tipo 2. Las complicaciones crónicas de la DM son las principales responsables de la morbidad y la mortalidad de los pacientes y, aunque no existan datos poblacionales sobre su prevalencia en Brasil, se estima que el número de ellas sea elevado. Además de eso, probablemente solo una pequeña fracción de la población de los pacientes diabéticos es evaluada regularmente por la presencia de complicaciones en sus fases iniciales y recibe orientaciones terapéuticas apropiadas ${ }^{(29)}$.

El presente estudio mostró que los entrevistados tienen acceso a las orientaciones sobre la DM y un alto por ciento de adhesión (el 98,0\%) al tratamiento médico para la DM. Se percibió, también, casi unánimemente en cuanto a ese punto, confirmando así los estudios realizados por varios investigadores, que relataron un aumento de la adhesión del tratamiento para la DM entre los pacientes atendidos en el programa Estrategia Salud de la Familia ${ }^{(30)}$.

El proceso de envejecimiento afecta a todos los componentes del control de la postura, sensorial, realizador y de procesamiento central, lo que también se refleja directamente en las habilidades del individuo para realizar las tareas cotidianas, o sea, en su capacidad funcional.

Se define la incapacidad funcional como la ausencia de habilidad o la dificultad para la realización de las tareas que forman parte de la cotidianeidad del individuo y que normalmente son indispensables para una vida independente en comunidad. Mientras que la capacidad funcional se refiere a la potencialidad para desempeñar las AVD o para realizar determinado acto sin ayuda para proporcionar una mejor calidad de vida $^{(31)}$.

La evaluación de la capacidad funcional es fundamental para determinar el compromiso y la necesidad de auxilio para las actividades de manutención y promoción de la propia salud y de gestión del ambiente domiciliario por parte de los ancianos, pudiendo guiar las políticas públicas de atención a la salud y las políticas sociales para este segmento ${ }^{(7)}$.

En el presente estudio, se observó una prevalancia de un $79,8 \%$ de alguna dependencia de los participantes, dato alarmante. En tal sentido, el proceso de envejecimiento provoca el declive de la aptitud física y de la capacidad funcional, convirtiendo a los ancianos en dependientes de los cuidados de otras personas. Por eso, la evaluación de la capacidad funcional y la aptitud física de los ancianos para orientar intervenciones específicas y su acompañamiento constante resultan fundamentales en el combate de las dependencias previsibles y en la promoción de una vida lo más activa posible ${ }^{(31)}$. En otro análisis, sus autores afirman que los aspectos de relaciones sociales tales como: "el no visitar a los parientes y/o los amigos por lo menos una vez a la semana", " el no participar de alguna obra social" y "el no participar de eventos sociales" se destacaron significativamente en el deterioro de la capacidad funcional ${ }^{(32)}$.

El desarrollo del cuidado gerontológico del anciano con diabetes demanda una necesidad de interacción, a fin de comprender y captar el modo de vivir de estos, así como de sus familiares y/o individuos involucrados en este proceso ${ }^{(33-34)}$. 


\section{CONCLUSIÓN}

Los resultados de la presente investigación mostraron que con relación a las condiciones clínicas, el 94,9\% refirieron co-morbidades; el 54,5\% presentaron complicaciones de diabetes; un $84,8 \%$ relataron sentir dolor en por lo menos una parte del cuerpo; el $78 \%$ utilizaban algún tipo de prótesis/órtesis; el $26,3 \%$ y un $22,2 \%$ informaron ingreso y caídas en el último año, respectivamente. Referente a las condiciones de funcionalidad, el $59,6 \%$ de los encuestados relataron problemas geriátricos, siendo que un 9,1\% referieron inmovilidad parcial; el 30,3\% inestabilidad de postura, el $5,1 \%$ insuficiencia cognitiva; el $28,3 \%$ incontinencia urinaria y el $23,3 \%$ presentan dificultades en la realización de las Actividades de la Vida Diaria.

En cuanto al grado de dependencia, se evidenció que la prevalencia de algún nivel de dependencia en los ancianos entrevistados es alta, del $(79,8 \%)$, siendo el $40,4 \%$ para dependencia leve; un $20,2 \%$ para moderada y un $19,2 \%$ para grave, demostrando la magnitud de las repercusiones de la DM tipo2 en la capacidad funcional para las AVD. Condición que, conforme ya fue descrito, fue ratificada por los propios entrevistados.

Los resultados llaman la atención y sugieren que los profesionales de la Atención Básica a la Salud vigilen y aseguren el uso de tecnologías leves, como las escalas de evalución de AVD y exámenes clínicos periódicos de estas actividades, para dirigir y adoptar medidas eficaces para el control y la optimización de la funcionalidad de los ancianos, ya que las informaciones sobre las condiciones de vida y de salud de ese grupo poblacional representan importantes aportes para el gerenciamiento de los cuidados de la salud de la población anciana-, contribuyendo así para la disminución de las vulnerabilidades, el mantenimiento de la salud y la protección contra los problemas precoces de las comorbidades en los ancianos diabéticos.

En ese contexto, la capacitación técnica del equipo es de fundamental importancia con relación a la atención de las necesidades específicas de los ancianos a la funcionalidad excelente, la prevención de las caídas, los cuidados con la piel, la incontinencia urinaria, así como también las medidas para la atención global de la salud de esta población.

Por fin, se verificó y se confirmó con este estudio la relación que existe entre el déficit en la capacidad funcional y la diabetes y el envejecimiento. Por ello, este trabajo cobra una gran relevancia para la Atención Básica a la Salud, toda vez que permitió la identificación de las incapacidades funcionales de los ancianos encuestados y las posibles lagunas en el acompañamiento/monitoría de estos, dotando al NASF investigado para el desarrollo de estrategias y así promover la prevención de las incapacidades y la prescripción de los cuidados dirigidos para las demandas funcionales específicas de la población anciana

\section{REFERENCIAS}

1. Fundo de População das Nações Unidas (UNFPA). Relatório sobre a situação da população Mundial, 2011 [acesso em 2012 nov 22]. Disponível em: http://www.un.cv/files/PT-SWOP11-WEB.pdf

2. Instituto Brasileiro de Geografia e Estatística. Censo 2010, 2010 [acesso em 2012 nov 22]. Disponível em: www.ibge.gov.br

3. Curpenito APFP, Rosa FHM, Ribeiro PCC. Definição de envelhecimento saudável na perspectiva de indivíduos idosos. Psicol reflex crit. 2007;20(1):81-86. 
4. Tavares DMS, Pereira GA, Iwamoto HH, Miranzzi SSC, Rodriques LR, Machado ARM. Incapacidade funcional entrei dosos residentes em um município do Interior de Minas Gerais. Texto contexto enferm. 2007;16(1):32-9.

5. Parahyba MI, Veras R, Melzer D. Incapacidade funcional entre mulheres idosas no Brasil. Rev Saúde Pública. 2005;39(3):383-91.

6. Ramos LR. Fatores determinantes do envelhecimento saudável em idosos residentes em centro urbano: Projeto Epidoso. Cad saúde pública. 2003; 19(3):793-798.

7. Nunes DP, Nakatani AYK, Silveira, Bachion MM, Souza MR. Capacidade funcional, condições socioeconômicas e de saúde de idosos atendidos por equipes de Saúde da Família de Goiânia (GO, Brasil). Ciên Saúde Coletiva. 2010;15(6):2887-2898.

8. Oliveira FC, Campos ACS, Alves MDS. Autocuidado do nefropata diabético. Rev Bras Enferm. 2010; 63(6):946-949.

9. Cardoso JH, Costa JSD. Características epidemiológicas, capacidade funcional e fatores associados em idosos de um plano de saúde. Ciênc saúde coletiva. 2010;15(6):2871-2878.

10. Giacomin KC, Peixoto SV, Uchoa E, Lima-Costa MF. Estudo de base populacional dos fatores associados à incapacidade funcional entre idosos na Região Metropolitana de Belo Horizonte, Minas Gerais, Brasil. Cad. Saúde Pública. 2008; 24(6):1260-1270.

11. Grillo MFF, Gorini MIPC. Caracterização de pessoas com Diabetes Mellitus Tipo 2. Rev Bras Enferm. 2007;60(1):49-64.

12. Gold SM, Dziobek I, Sweat V, Tirsi A, Rogers K, Bruehl H, et al. Hippocampal damage and memory impairments as possible early brain complications of type 2 diabetes. Diabetologia. 2007; 50(4):711-719.

13. Alvarenga PP, Pereira DSP, Anjos DMC. Mobilidade funcional e função executiva em idosos diabéticos e não diabéticos. Rev Bras Fisioter. 2010;14(6):491-496.

14. Stacciarini TSG, Haas VJ, Pace AE. Fatores associados à auto-aplicação da insulina nos usuários com diabetes mellitus acompanhados pela Estratégia Saúde da Família. Cad Saúde Pública. 2008;24 (6):1314-1322.

15. Francisco PMSB, Belon AP, Barros MBA, Carandina L, Alves MCGP, Goldbaum M et al. Diabetes auto-referido em idosos: prevalência, fatores associados e práticas de controle. Cad Saúde Pública. 2010;26(1):175-184.

16. Lima-Costa MFF, Matos DL, Camarano AA. Evolução das desigualdades sociais entre idosos e adultos brasileiros: um estudo baseado na Pesquisa Nacional por Amostras de Domicílio (PNAD, 1998, 2003). Ciênc Saúde Coletiva. 2006;11(1):941-50.

17. Nunes MCR, Ribeiro RCL, Rosado LEFPL, Franceschini SC. Influência das características sóciodemográficas e epidemiológicas na capacidade funcional de idosos residentes em Ubá, Minas Gerais. Rev Bras Fisioter. 2009; 13 (5): 376-382.

18. Moreira RO, Amâncio APRL, Brum HR, Vasconcelos DL, Nascimento GF. Sintomas depressivos e qualidade de vida em pacientes diabéticos tipo $2 \mathrm{com}$ polineuropatia distal diabética. Arq Bras Endocrinol Metab. 2009;53(9):1103-1111.

19. Ziegler D. Treatment of diabetic neuropathy and neuropathic pain: how far have we come. Diabetes Care. 2008;31(31Supl 2):255-261.

20. Loyola Filho AI, Uchoa E, Lima-Costa MF. Estudo epidemiológico de base populacional sobre uso de medicamentos entre idosos na Região Metropolitana de Belo Horizonte, Minas Gerais, Brasil. Cad Saúde Pública. 2006;22(12):2657-2667.

21. Silva EMM, Gallo AKG, Santos DM. Enfermidades do Paciente Idoso. Pesq Bras Odontoped Clin Integr. 2007;7(1):83-88.

22. Higa R, Lopes MHBM, Reis MJ. Fatores de risco para incontinência urinária na mulher. Rev Esc Enferm USP. 2008;42(1):187-192.

23. Kagansky $\mathrm{N}$, Levy $\mathrm{S}$, Knobler $\mathrm{H}$. The role of hyperglycemia in acute stroke. Arch Neurol. 2001;58(8):1209-1212. 
24. Blaum CS, Ofstedal MB, Liang JL. Cognitive Performance, Comorbid Disease, and Task-Specific Disability: Findings from a Nationally Representative Survey. Jour Gerontol. 2002;57(57 Suppl 8):523-531.

25. Tavares DMS, Pereira GA, Iwamoto HH, Miranzzi SSC, Rodrigues LR, Machado ARM. Incapacidade funcional entre idosos residentes em um município do interior de Minas Gerais. Texto contexto enferm. 2007;16(1):32-39.

26. Mendes TAB, Goldbaum M, Segri NJ, Barros MBA, Cesar CLG, Carandina L. Diabetes mellitus: fatores associados à prevalência em idosos, medidas e práticas de controle e uso dos serviços de saúde em São Paulo, Brasil. Cad Saúde Pública. 2011;27(6):1233-1243.

27. Silva LMC, Palha PF, Barbosa GR, Protti ST, Ramos AS. Aposentados com diabetes tipo 2 na Saúde da Família em Ribeirão Preto, São Paulo - Brasil. Rev Esc Enferm USP. 2010;44(2):462-468.

28. Moraes AS, Freitas ICM, Gimeno SGA, Mondini L. Prevalência de diabetes mellitus e identificação de fatores associados em adultos residentes em área urbana de Ribeirão Preto, São Paulo, Brasil, 2006: Projeto OBEDIARP. Cad Saúde Pública. 2010;26(5):929-941.

29. Guedes MF, Portes AJF, Couto JAS, Nunes JS, Oliveira RCC. Prevalência da retinopatia diabética em unidade do Programa de Saúde da Família. Rev bras oftalmol. 2009;68(2):90-95.

30. Santos FS, Oliveira KR, Colet CF. Adesão ao tratamento medicamentoso pelos portadores de Diabetes Mellitus atendidos em uma Unidade Básica de Saúde no município de ljuí/RS: um estudo exploratório. Rev Ciênc Farm Básica Apl. 2010;31(3):223-227.

31. Gonçalves LHT, Silva AH, Mazo GZ, Benedetti TRB, Santos SMA, Marques S. O idoso institucionalizado: avaliação da capacidade funcional e aptidão física. Cad Saúde Pública. 2010;26(9):1738-1746.

32. Nogueira SL, Ribeiro RCL, Rosado LEFPL, Franceschini SCC, Ribeiro AQ, Pereira ET. Fatores determinantes da capacidade funcional em idosos longevos. Rev Bras Fisioter. 2010;14(4):322-329.

33. Hammerschmidt KSA, Lenardt $\mathrm{MH}$. Tecnologia educacional inovadora para $\mathrm{O}$ empoderamento junto a idosos com diabetes mellitus. Texto contexto enferm. 2010;19(2):358-365.

34. Tavares DMS, Rodrigues RAP. Indicadores sócio-demográficos e de saúde de idosos portadores e não portadores de diabetes. Rev Elet Enferm [periódico online]. 2008 [acesso em 2013 jan 22];10(4):906-914. Disponível em: http://www.fen.ufg.br/revista/v10/n4/v10n4a03.htm 